\title{
Accurate Motion Parameter Estimation For Colonoscopy Tracking Using a Regression Method
}

\author{
Jianfei Liu ${ }^{a}$ and Kalpathi R. Subramanian ${ }^{a}$ Terry S. Yoo ${ }^{b}$ \\ ${ }^{a}$ The University of North Carolina at Charlotte \\ Charlotte Visualization Center, Dept. of Computer Science, \\ 9201 University City Blvd, Charlotte, North Carolina, USA \\ ${ }^{b}$ Office of High Performance Computing and Communications, \\ National Library of Medicine, National Institutes of Health, Bethesda, Maryland, USA
}

\begin{abstract}
Co-located optical and virtual colonoscopy images have the potential to provide important clinical information during routine colonoscopy procedures. In our earlier work, we presented an optical flow based algorithm to compute egomotion from live colonoscopy video, permitting navigation and visualization of the corresponding patient anatomy. In the original algorithm, motion parameters were estimated using the traditional Least Sum of squares(LS) procedure which can be unstable in the context of optical flow vectors with large errors. In the improved algorithm, we use the Least Median of Squares (LMS) method, a robust regression method for motion parameter estimation. Using the LMS method, we iteratively analyze and converge toward the main distribution of the flow vectors, while disregarding outliers. We show through three experiments the improvement in tracking results obtained using the LMS method, in comparison to the LS estimator. The first experiment demonstrates better spatial accuracy in positioning the virtual camera in the sigmoid colon. The second and third experiments demonstrate the robustness of this estimator, resulting in longer tracked sequences: from 300 to 1310 in the ascending colon, and 410 to 1316 in the transverse colon.
\end{abstract}

Keywords: Colonoscopy tracking, optical flow, regression, egomotion

\section{INTRODUCTION}

Colorectal cancer is the third most common cancer and a leading cause of cancer-related mortality in the United States. ${ }^{1}$ Currently, two technologies exist for colon screening, (1) Optical Colonoscopy(OC), an exploratory procedure to detect and treat colon cancer, and, (2) Virtual Colonoscopy(VC), ${ }^{2,3}$ a screening only tool based on CT images, and capable of providing 3D interactive views of the colon. There are tradeoffs with both approaches: OC can miss polyps, relies on physician skills and experience, while VC cannot detect lesions less than $5 \mathrm{~mm}$. The goal of our work is to investigate methods to automatically and accurately track presegmented VC images during an OC procedure, so as to maximize the useful information presented to the physician, and minimize errors. Fig. 1 illustrates the overall goal of this research.

A number of researchers have focused on tracking bronchoscopy images. ${ }^{4-10}$ These methods are based on image registration, and exploit anatomical marks like bifurcations and structural features that characterize the bronchia. Bricault et al. ${ }^{4}$ proposed a multi-stage tracking algorithm to align optical and virtual images. Rai et al. ${ }^{5}$ applied pose estimation to match virtual and optical images, assuming similarity in depth values. Deguchi et al. ${ }^{6}$ utilized sum-of-square difference as a cost metric to measure the similarity of intensity distribution between virtual and optical images, in order to estimate camera motion. In the context of image guided navigation, we proposed an optical flow based approach for tracking colonoscopy images. ${ }^{11}$

Further author information: (Send correspondence to Kalpathi R. Subramanian)

Jianfei Liu: E-mail: jliu1@uncc.edu

Kalpathi R. Subramanian: E-mail: krs@uncc.edu

Terry S. Yoo: E-mail:yoo@nlm.nih.gov

Medical Imaging 2010: Computer-Aided Diagnosis, edited by Nico Karssemeijer, Ronald M. Summers, Proc. of SPIE Vol. 7624,76241 Y · C 2010 SPIE · CCC code: 1605-7422/10/\$18 · doi: 10.1117/12.844433 


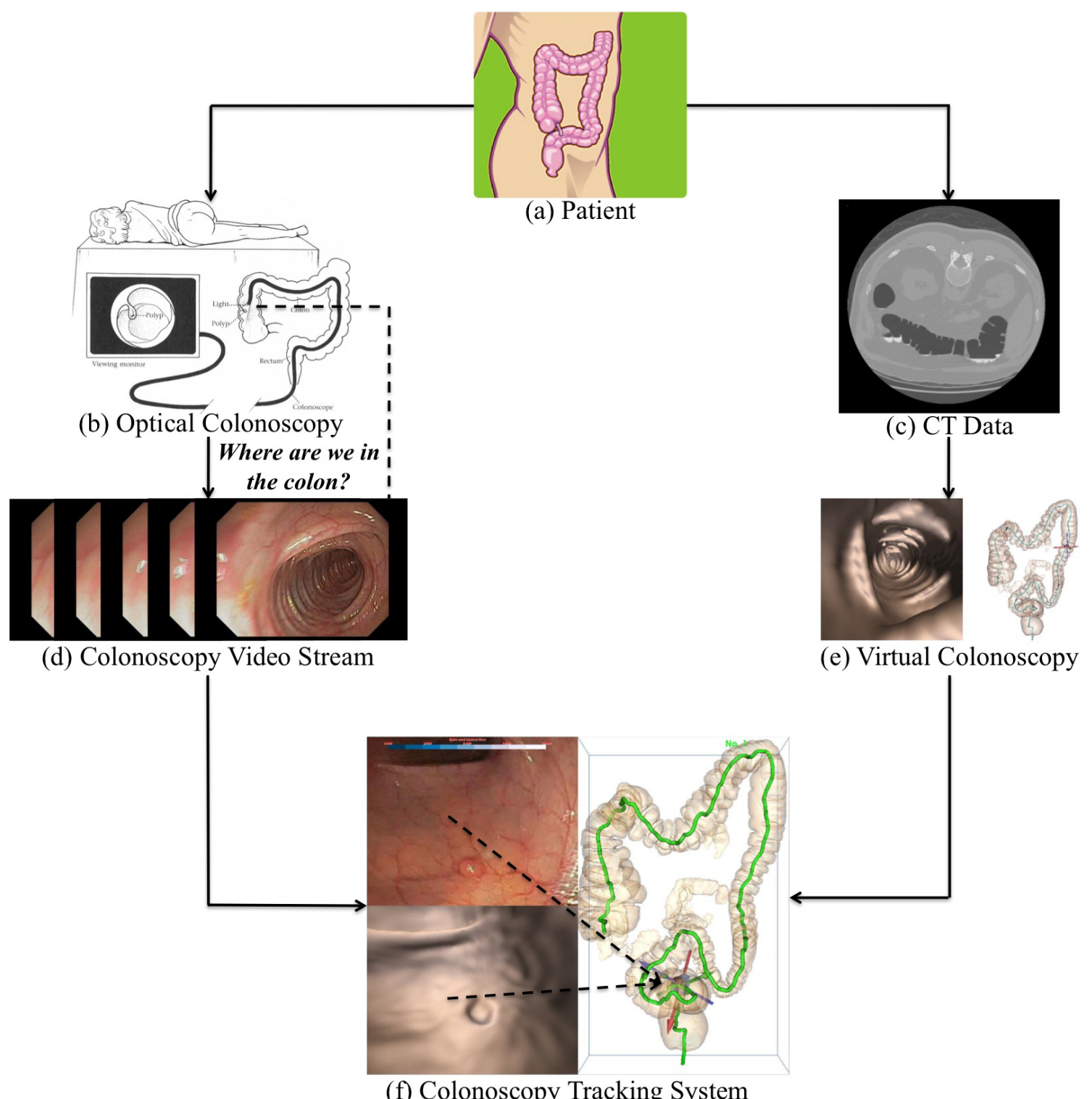

Figure 1. Colonoscopy Tracking. Optical colonoscopy images(d) and reconstructed CT images(e) are co-located and presented to the physician during a routine colonoscopy procedure(f). Such a visualization is clinically useful in providing a better spatial perspective, especially in the vicinity of important features, such as polyps. ((a) and (b) reproduced from http://www.answers.com/topic/colonoscopy.)

In most of these approaches, camera motion parameters were estimated using the Least Sum of squares(LS) method; As indicated by Hampel ${ }^{12}$ and Rousseeuw et al., ${ }^{13}$ LS is sensitive to outliers and a robust regression method is needed to detect and remove them. Meer et al. ${ }^{14}$ reviewed different robust estimators like Mestimators ${ }^{12}$ to compute planar surface patches. Stewart ${ }^{15}$ extended this review and illustrated application to fundamental matrix estimation, surface reconstruction, and construction of retinal image mosaics. Black et al. ${ }^{16}$ applied Geman \& McClure ${ }^{12}$ and Lorentzian ${ }^{12}$ estimators to identify motion discontinuities in prevention of the smoothness constraint violation in optical flow computation. Zhang et al. ${ }^{17}$ investigated the least median of squares method to accurately estimate epipolar geometry. Similar work was done by Hartley and Zisserman, ${ }^{18}$ however they used the RANSAC (RANdom SAmple Consensus) ${ }^{19}$ estimator. 
Optical flow computation in colonoscopy images is subject to errors, requiring robust methods for motion parameter estimation. Occlusion effects between succeeding frames can result in unstable optical flow vectors in the obstructed areas. Even a few vectors with large errors could result in a tracking failure. In this paper, we improve the accuracy and robustness of the motion parameter computation by integrating the Least Median of Squares (LMS) estimator with our current tracking algorithm ${ }^{11}$ to detect these outliers. Sec. 2 describes the proposed method. A series of 3 experiments on clinical colonoscopy sequences are presented in Sec. 3. Section 4 summarizes our work.

\section{METHODS}

We will first briefly describe our current colonoscopy tracking algorithm. ${ }^{11}$

\subsection{Colonoscopy Tracking Algorithm}

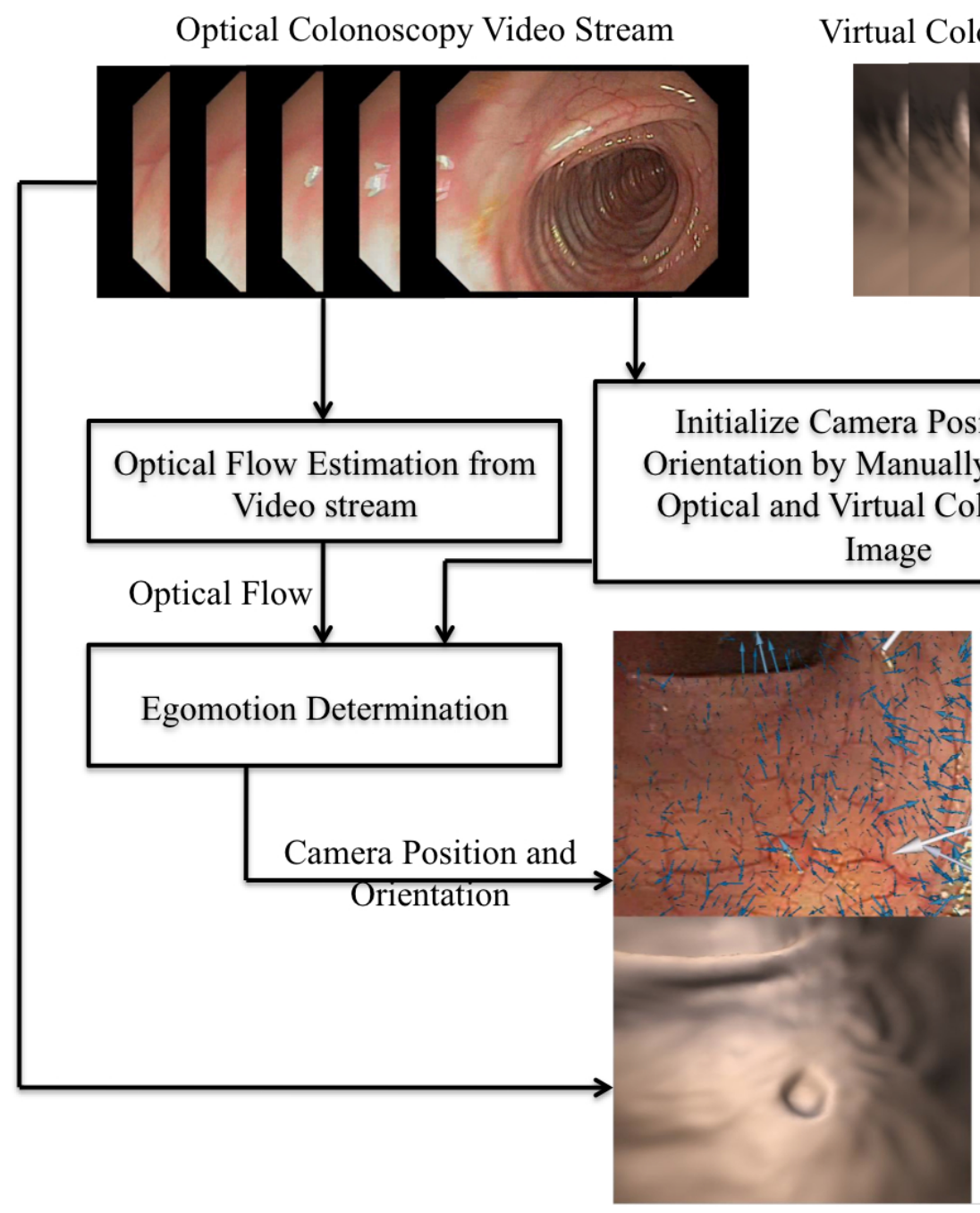

irtual Colonoscopy Video Stream
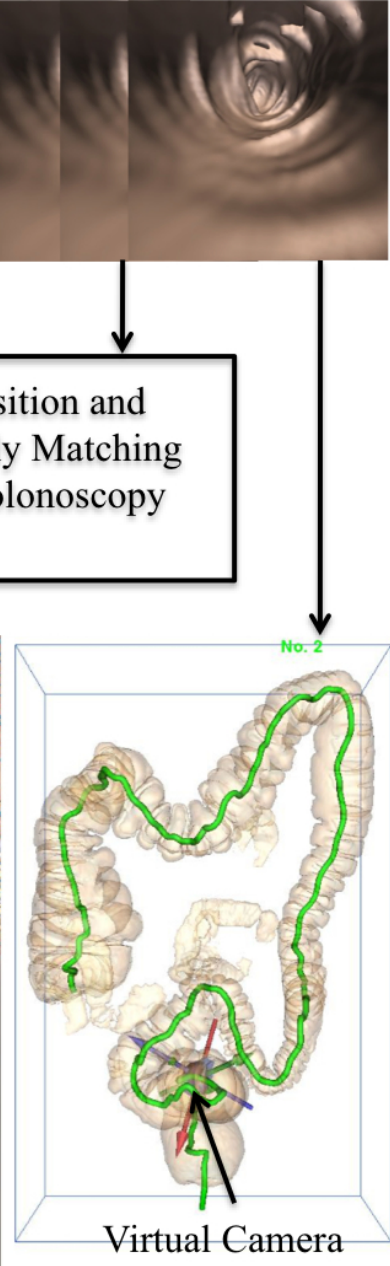

Figure 2. The colonoscopy Tracking Algorithm.

Fig. 2 illustrates the colonoscopy tracking algorithm. The camera position and orientation are first initialized through manually adjusting optical and virtual colonoscopy images. A multi-scale strategy is used to compute sparse and dense optical flow fields in each optical image. The sparse flow field is then used to determine 
camera motion parameters with the help of the Focus of Expansion(FOE), which is estimated from dense optical flow. Optical and virtual colonoscopy are finally aligned through transforming the estimated camera motion parameters. Complete details of the algorithm can be found in our earlier work. ${ }^{11}$

\subsection{Motion Parameter Estimation}

Next we describe motion parameter computation in more detail. Assume $\vec{T}=\left(T_{x}, T_{y}, T_{z}\right)$ and $\vec{R}=\left(R_{x}, R_{y}, R_{z}\right)$ represent the translation and rotation velocities of the optical colonoscope. Let an object point $P$ in camera coordinate be $(X, Y, Z)$ and its projection point $p$ in the image plane be $(x, y)$. Assuming focal length $f=1$, the optical flow vector $20 \vec{u}=\left(u_{x}, u_{y}\right)$ at $p$ is given by

$$
\begin{aligned}
& u_{x}=\frac{T_{z}}{Z}\left(x-\frac{T_{x}}{T_{z}}\right)+R_{x} x y-R_{y}\left(1+x^{2}\right)+R_{z} y \\
& u_{y}=\frac{T_{z}}{Z}\left(y-\frac{T_{y}}{T_{z}}\right)+R_{x}\left(1+y^{2}\right)-R_{y} x y-R_{z} x
\end{aligned}
$$

We can split Eq. 1 into translation and rotation components, thus

$$
\vec{u}=\vec{u}^{T}+\vec{u}^{R}
$$

where $\vec{u}^{T}=\left(u_{x}^{T}, u_{y}^{T}\right)$ and $\vec{u}^{R}=\left(u_{x}^{R}, u_{y}^{R}\right)$ and

$$
\begin{aligned}
u_{x}^{T}=\frac{T_{z}}{Z}\left(x-\frac{T_{x}}{T_{z}}\right) & u_{y}^{T}=\frac{T_{z}}{Z}\left(y-\frac{T_{y}}{T_{z}}\right) \\
u_{x}^{R}=R_{x} x y-R_{y}\left(1+x^{2}\right)+R_{z} y & u_{y}^{R}=R_{x}\left(1+y^{2}\right)-R_{y} x y-R_{z} x
\end{aligned}
$$

The translation component can be eliminated by transforming the optical flow into polar coordinates, with the focus of expansion $\left(\frac{T_{x}}{T_{z}}, \frac{T_{y}}{T_{z}}\right)$ at the center, as follows:

$$
\vec{u} \cdot \vec{e}_{\perp}=\left(\vec{u}^{T}+\vec{u}^{R}\right) \cdot \vec{e}_{\perp}=\vec{u}^{R} \cdot \vec{e}_{\perp}
$$

where $\vec{e}_{\perp}$ is perpendicular to the translation component $\vec{u}^{T}$. Substituting Eq. 4 into Eq. 5 , the estimation of rotation parameters can be converted into the following linear system:

$$
\left\{\begin{array}{c}
\left(x_{1} y_{1} e_{\perp x_{1}}+\left(y_{1}^{2}+1\right) e_{\perp y_{1}}\right) R_{x}-\left(\left(x_{1}^{2}+1\right) e_{\perp x_{0}}+x_{1} y_{1} e_{\perp y_{1}}\right) R_{y}+\left(y_{1} e_{\perp x_{1}}-x_{1} e_{\perp y_{1}}\right) R_{z}=\mathbf{u}_{\mathbf{1}} \cdot \mathbf{e}_{\perp_{\mathbf{1}}} \\
\vdots \\
\left(x_{n} y_{n} e_{\perp x_{n}}+\left(y_{n}^{2}+1\right) e_{\perp y_{n}}\right) R_{x}-\left(\left(x_{n}^{2}+1\right) e_{\perp x_{n}}+x_{n} y_{n} e_{\perp y_{n}}\right) R_{y}+\left(y_{n} e_{\perp x_{n}}-x_{n} e_{\perp y_{n}}\right) R_{z}=\mathbf{u}_{\mathbf{n}} \cdot \mathbf{e}_{\perp_{\mathbf{n}}}
\end{array}\right.
$$

where $n$ is the number of sparse optical flow vectors and $\left(x_{i}, y_{i}\right), i \in(1, n)$, is the $i$ th feature point in the image plane.

To compute the translation parameters $\vec{T}$, substitute the estimated rotation parameters into Eqs. 1, followed by $\min _{\vec{T}}\left(\vec{u}^{T}-\left(\vec{u}-\vec{u}^{R}\right)\right)^{2}$. This results in groups of three linear equations for each feature point, as shown below

$$
\left\{\begin{array}{c}
\left(\begin{array}{ccc}
-\frac{1}{Z_{1}} & 0 & \frac{x_{1}}{Z_{1}} \\
0 & -\frac{1}{Z_{1}} & \frac{y_{1}}{Z_{1}} \\
-\frac{x_{1}}{Z_{1}} & -\frac{y_{1}}{Z_{1}} & \frac{x_{1}^{2}+y_{1}^{2}}{Z_{1}}
\end{array}\right)\left(\begin{array}{c}
T_{x} \\
T_{y} \\
T_{z}
\end{array}\right)= \\
\left(\begin{array}{ccc}
-\frac{1}{Z_{n}} & 0 & \frac{x_{n}}{Z_{n}} \\
0 & -\frac{1}{Z_{n}} & \frac{y_{n}}{Z_{n}} \\
-\frac{x_{n}}{Z_{n}} & -\frac{y_{n}}{Z_{n}} & \frac{x_{n}^{2}+y_{n}^{2}}{Z_{n}}
\end{array}\right)\left(\begin{array}{c}
T_{x} \\
T_{y} \\
T_{z}
\end{array}\right)=\left(\begin{array}{c}
u_{x 1}-u_{x 1}^{R} \\
u_{y 1}-u_{y 1}^{R} \\
x_{1}\left(u_{x 1}-u_{x 1}^{R}\right)+y_{1}\left(u_{y 1}-u_{y 1}^{R}\right)
\end{array}\right) \\
\vdots
\end{array}\right.
$$

where $Z_{i}$ is the depth value of the current selected feature point from $Z$-buffer. 


\subsection{Least Median of Squares (LMS) Estimation}

This section briefly introduces some basic concepts of LMS estimation, as described in Rousseeuw. ${ }^{13}$ The classical linear model can be represented as

$$
y_{i}=x_{i 1} \theta_{1}+\cdots+x_{i p} \theta_{p}+e_{i} \text { for } i=1, \ldots, m,
$$

where $m$ is the sample size. The variables $x_{i 1}, \ldots, x_{i p}$ are called the explanatory variables, whereas the variable $y_{i}$ is called the response variable. $e_{i}$ is the error term, assumed to be a normal distribution. The aim of linear regression is to estimate $\theta=\left[\theta_{1}, \cdots, \theta_{p}\right]^{T}$. Applying a regression technique like the least sum of squares to yield $\hat{\theta}=\left[\hat{\theta}_{1}, \cdots, \hat{\theta}_{p}\right]^{T}$, the following formula can be obtained

$$
\hat{y}_{i}=x_{i 1} \hat{\theta}_{1}+\cdots+x_{i p} \hat{\theta}_{p}
$$

The residual $r_{i}$ of the $i$ th case is the difference between what is actually observed and what is estimated:

$$
r_{i}=y_{i}-\hat{y}_{i}
$$

In terms of Eq. 10, the least sum of squares can be expressed as

$$
\min _{\hat{\theta}} \sum_{i=1}^{m} r_{i}^{2}
$$

However, $e_{i}$ is not always a normal distribution and the LS estimator can be highly sensitive to outliers. Rousseeuw ${ }^{21}$ proposed the Least Median of Squares(LMS) to analyze the main distribution of the dataset to identify the outliers. LMS is defined as

$$
\min _{\hat{\theta}} \operatorname{med}_{i} r_{i}^{2}
$$

\subsection{LMS Based Colonoscopy Tracking Algorithm}

We first estimate the rotation parameters. This consists of 6 steps:

1. Randomly choose three linear equations from Eq. 6 to compute rotation parameters, corresponding to the unknown rotation parameters, $\vec{R}=\left(R_{x}, R_{y}, R_{z}\right)$.

2. Substitute $\vec{R}$ into Eq. 6 and determine the median of the squared residuals, $M_{j}$, where $j$ is the current iteration number.

$$
M_{j}=\operatorname{med}_{i \in[1, n]}\left(r_{i}^{R}\right)^{2}
$$

where $n$ is the number of sparse optical flow vectors, residual $r_{i}^{R}$ is the difference between what is actually observed and what is estimated in $i$ th equation of Eq. 6 .

3. Iterate steps 1 and $2 J$ times until all possible input data distributions are investigated and choose the minimum $M_{j}, j \in(1, K)$. Report the corresponding rotation, $\vec{R}$.

4. Compute the scale of the minimum residual ${ }^{13}$ as

$$
s=1.4826\left(1+\frac{5}{n-3}\right) \sqrt{\frac{\min _{\vec{R}}}{\operatorname{med}_{i}\left(r_{i}^{R}\right)^{2}}}
$$

where $1 / \Phi^{-1}(0.75) \approx 1.4826$ is an asymptotic correction factor for the case of normal errors and $\Phi$ denotes the cumulative distribution function of the standard normal distribution. More details can be found in Rousseeuw. ${ }^{13,21}$

5. The residuals are next normalized, $\left(r_{i}^{R}\right) / s$, and used to weight the $i$ th observation,

$$
w_{i}= \begin{cases}1 & \text { if }\left|r_{i}^{R}\right| \leq 2.5|s| \\ 0 & \text { otherwise }\end{cases}
$$


6. The final rotation parameters $\vec{R}$ can be recomputed according to reweighted least squares (RLS) regression.

$$
\min _{\vec{R}} \sum_{i=1}^{n} w_{i}\left(r_{i}^{R}\right)^{2}
$$

A procedure similar to the rotation parameter estimation is followed, except that the squared residuals $\left(r_{i}^{T}\right)^{2}$ is not from a single equation but from the sum of the residuals resulting from the three equations in each group in Eq. 7. Assuming $\overrightarrow{\hat{T}}=\left(\hat{T}_{x}, \hat{T}_{y}, \hat{T}_{z}\right)$ be the estimated translation velocities at a particular iteration, then $\left(r_{i}^{T}\right)^{2}$ is given by

$$
\begin{aligned}
\left(r_{i}^{T}\right)^{2} & =\left(u_{x i}-u_{x i}^{R}-\left(-\frac{1}{Z_{i}} \hat{T}_{x}+\frac{x_{i}}{Z_{i}} \hat{T}_{z}\right)\right)^{2} \\
& +\left(u_{y i}-u_{y i}^{R}-\left(-\frac{1}{Z_{i}} \hat{T}_{y}+\frac{y_{i}}{Z_{i}} \hat{T}_{z}\right)\right)^{2} \\
& +\left(x_{i}\left(u_{x i}-u_{x i}^{R}+y_{i}\left(u_{y i}-u_{y i}^{R}\right)-\left(-\frac{x_{i}}{Z_{i}} \hat{T}_{x}-\frac{y_{i}}{Z_{i}} \hat{T}_{y}+\frac{x_{i}^{2}+y_{i}^{2}}{Z_{i}} \hat{T}_{z}\right)\right)^{2}\right.
\end{aligned}
$$

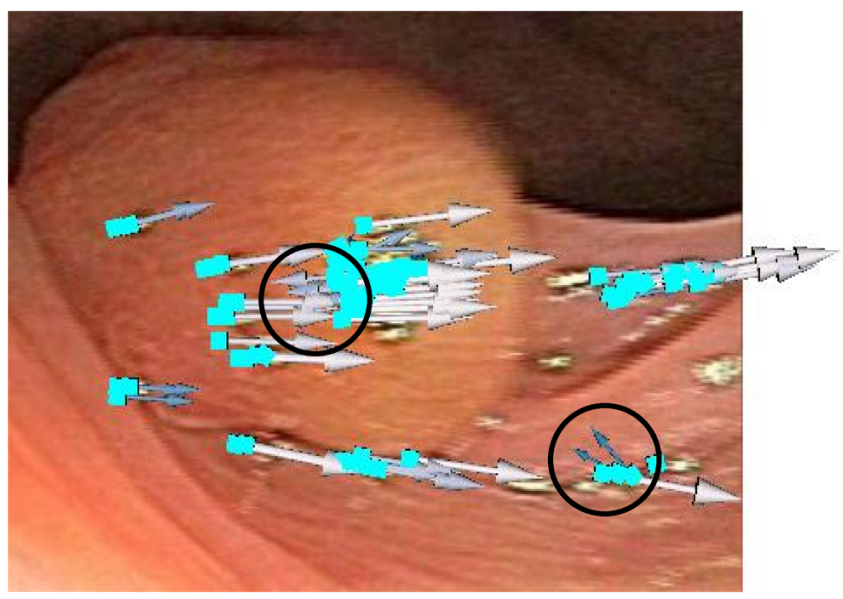

(a) Features selected by the LS method.

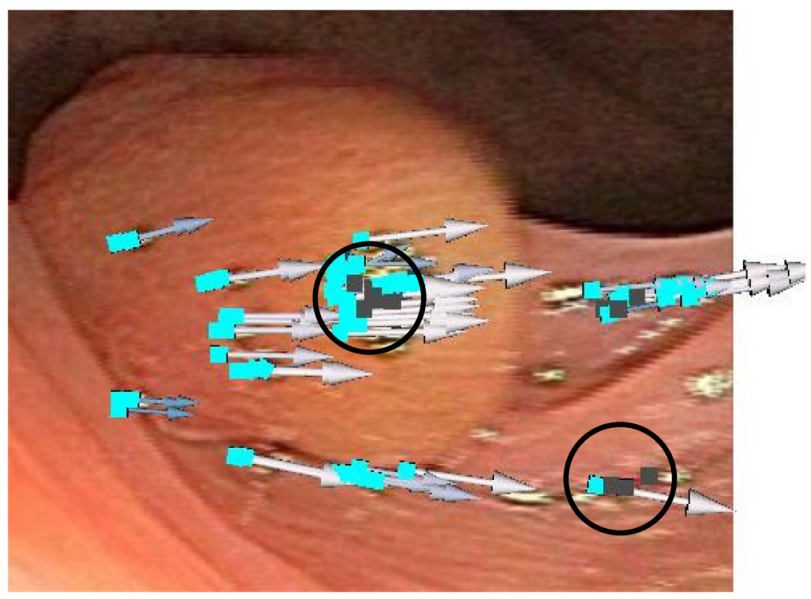

(b) Features selected by the LMS method.

Figure 3. Selected features (in cyan) used by the LS (a) and the LMS (b) methods on a tracked OC image in the transverse colon. Each cube represents the selected feature point. Black features are outliers and black circles indicate areas with outliers, where some feature vectors are pointing in the wrong direction (towards left), which is incorrect.

Fig. 3 shows the selected feature points of the LS and LMS methods within a particular optical image. Fig. 3(a) shows features(in cyan) used by the LS method. The right image illustrates the features used by the LMS method, where the marked features in black indicate outliers that are discarded. The black circles illustrate features that are wrongly directed and are detected as outliers.

\section{EXPERIMENTAL RESULTS}

This section illustrates three experiments using the LMS estimator and illustrates improvements to our tracking algorithm.

\subsection{Case 1: Sigmoid Colon}

The first sequence was captured in the sigmoid colon with the colonoscope moving around a polyp. The sequence has 320 frames. At frame 320, the colonoscope is close to the colon wall, and due to lack of features, the tracking algorithm stops. In this example, both the LS and LMS estimators are able to track the full sequence. Fig. 4 
Frame. 100

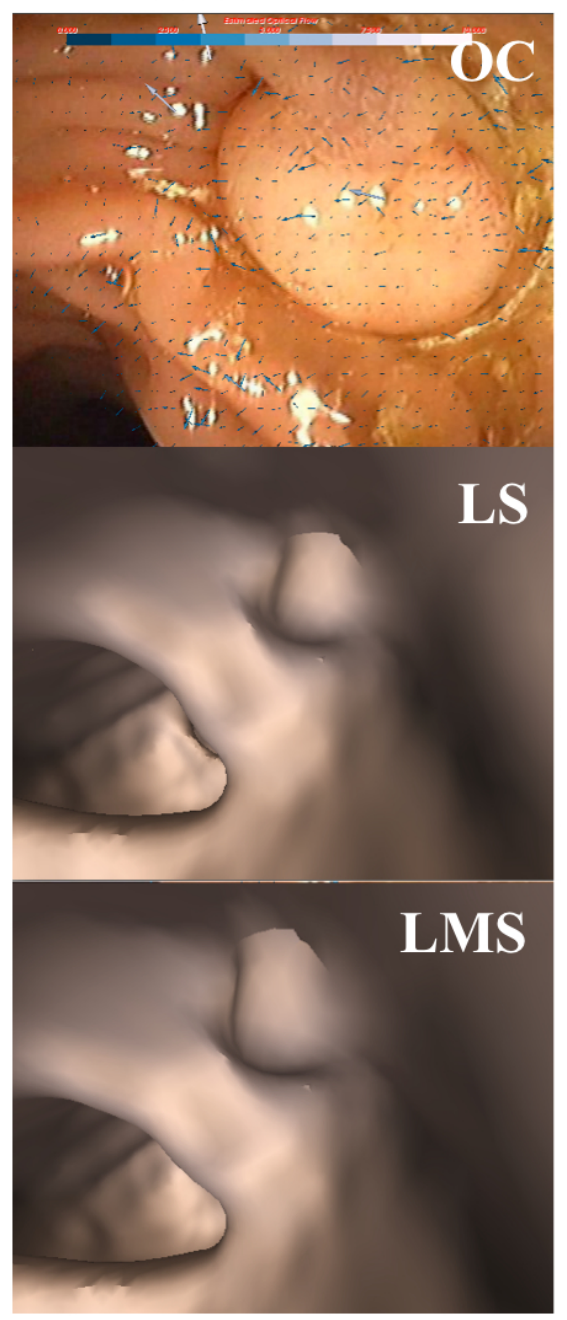

(a)
Frame. 200

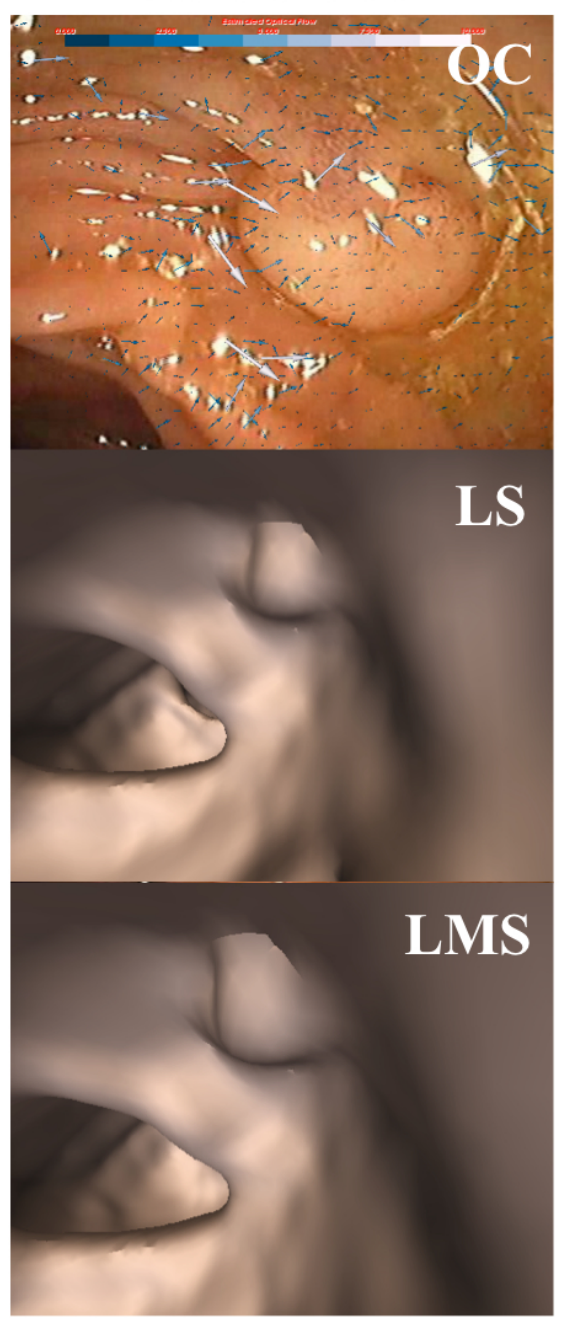

(b)
Frame. 276

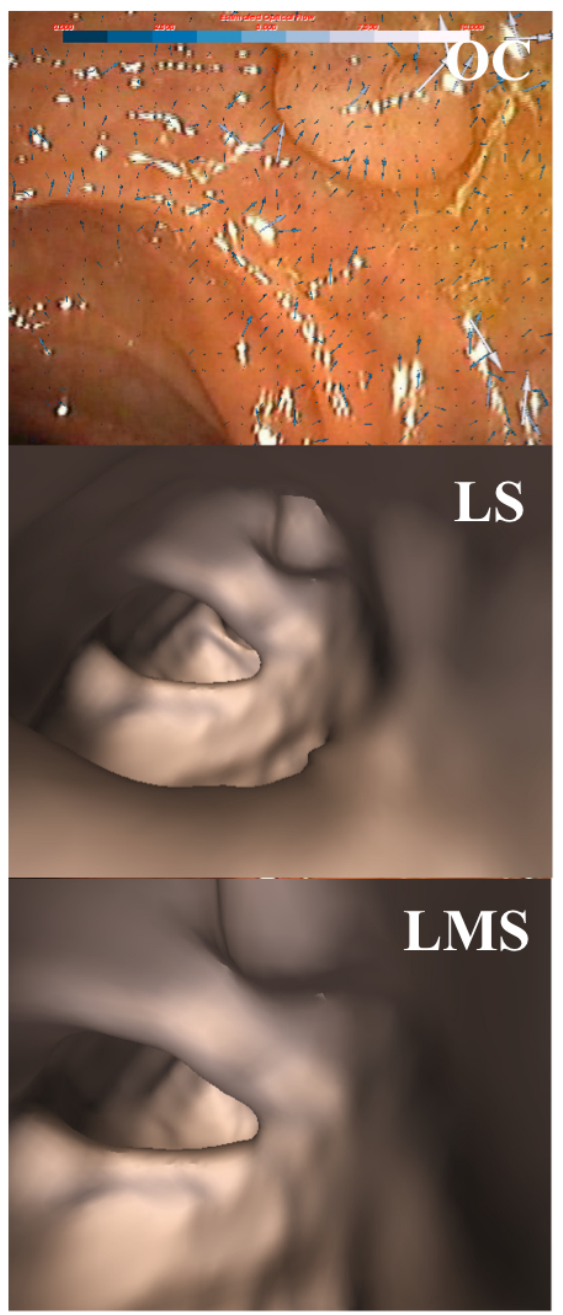

(c)

Figure 4. Comparison of LMS and LS tracking results in frame 100 (left column), 200 (center column), 276 (right column) in a sigmoid colon sequence. Top row shows the original optical images, and center and bottom rows illustrate the tracking results of LS and LMS estimators, respectively. LMS results are more accurate than those of the LS estimator as the size of virtual polyp is closer to the polyp in the optical image, and spatially more accurate(frame 276).

illustrates three snapshots at frames 100, 200, and 276. The top row shows the optical images, while the middle and bottom rows show the frames using the LS and LMS-based estimators. In frame 100 and 200, the LMS and LS images are similar, but the LMS images show the polyp to be somewhat larger. The accuracy of LMS is clearer in the images in the third column, as the LS image is off by the length of a fold, while the LMS-based image is still in the vicinity of the polyp.

\subsection{Case 2: Ascending Colon}

In this experiment, a sequence of 1310 images was acquired in the ascending colon with a flat polyp. Fig. 5 shows results at frames 100,136, and 300. The polyp is enclosed by a black circle. After frame 300, the virtual camera driven by the LS-based estimator approaches a colon fold and subsequently leaves the colon. The LMS-based estimator continues to track until the end of the sequence. Beyond frame 1310, surgical tool motion in the 
Frame. 100

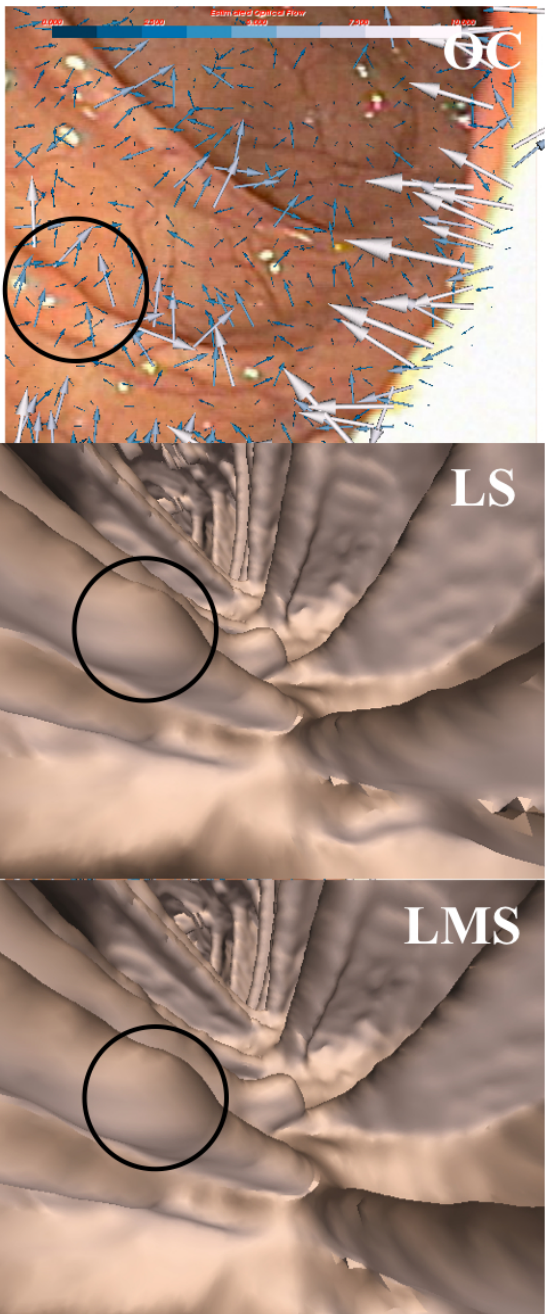

(a)
Frame. 136

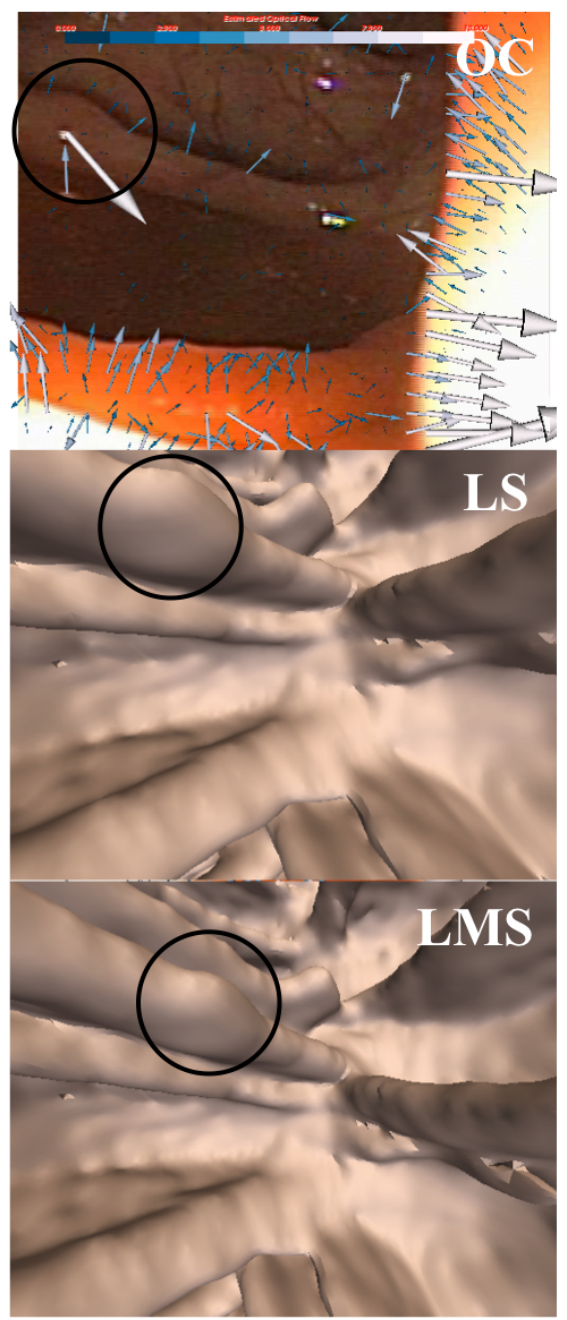

(b)
Frame. 300

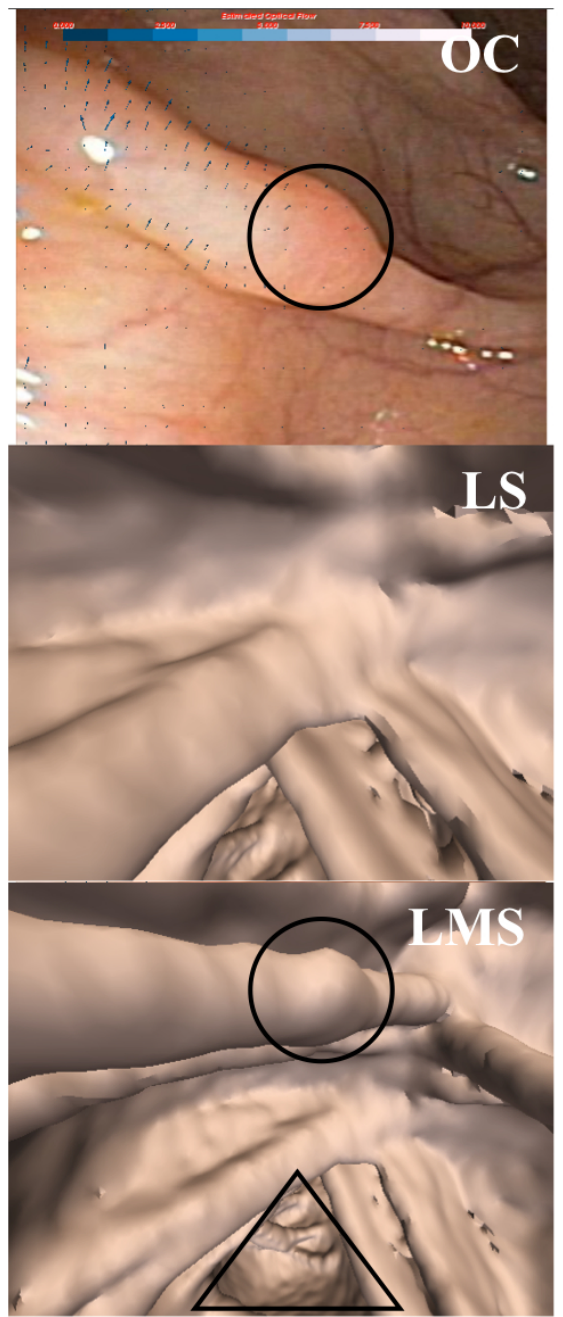

(c)

Figure 5. Comparison of LMS and LS tracking results in frame 100 (left column), 136 (center column), and 300 (right column) using an ascending colon image sequence of 1310 images. Here, the flat polyp is marked by a black circle. Due to shape differences(caused by deformation) between virtual and physical colon, a region marked by a black triangle appears in the virtual image, but not in the optical image. this is likely due to an artificial turn in the virtual colon.

images(polyp being surgically removed) stops the tracking. In frames 100 and 136, the LMS and LS methods closely follow the motion of colonoscope. However, the LS method accumulates errors after frame 136, due to several partially blurry images. The middle image, right column shows the tracking results at frame 300, where the virtual camera driven by the LS estimator tracks forward translation but not rotation, while the LMS results are more accurate for both. A fold marked by a black triangle appears in the tracked VC image in the right column, but is non-existent in the OC image. This is most likely due to an artificial turn in the virtual colon, but not the case during the surgical procedure.

\subsection{Case 3: Transverse Colon}

In this experiment, a sequence of 1316 images was acquired in the transverse colon with a polyp. At the end of the sequence, surgical tools appear in the images, which stops the tracking. As in experiment 2, a similar failure 


\section{Frame. 50}

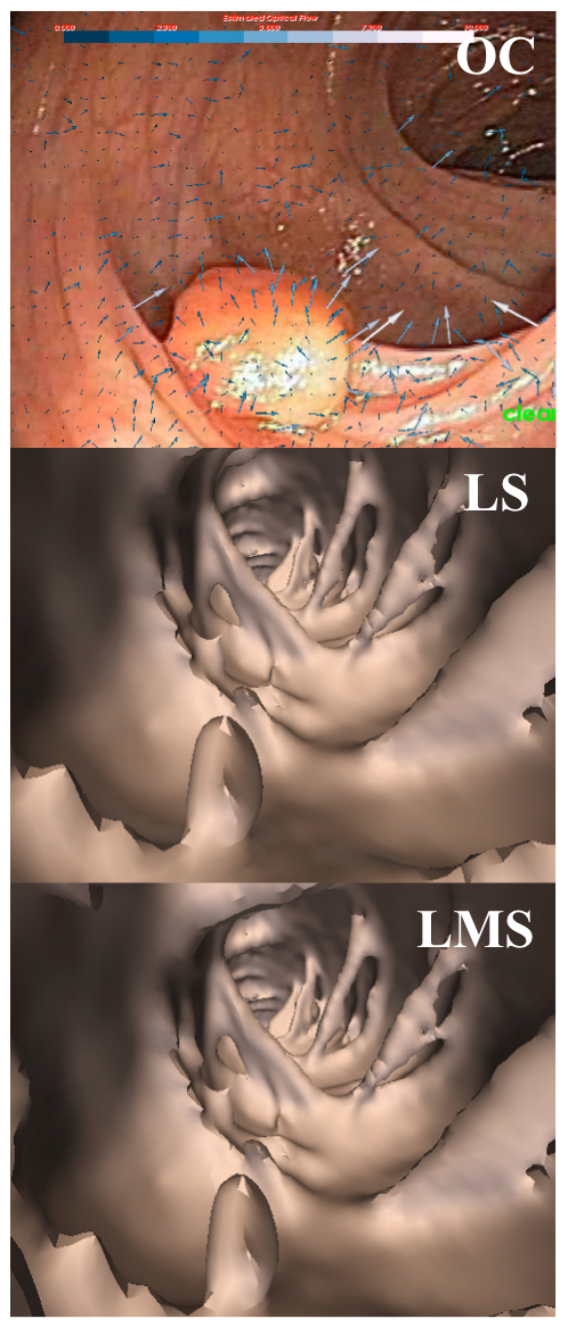

(a)
Frame. 240

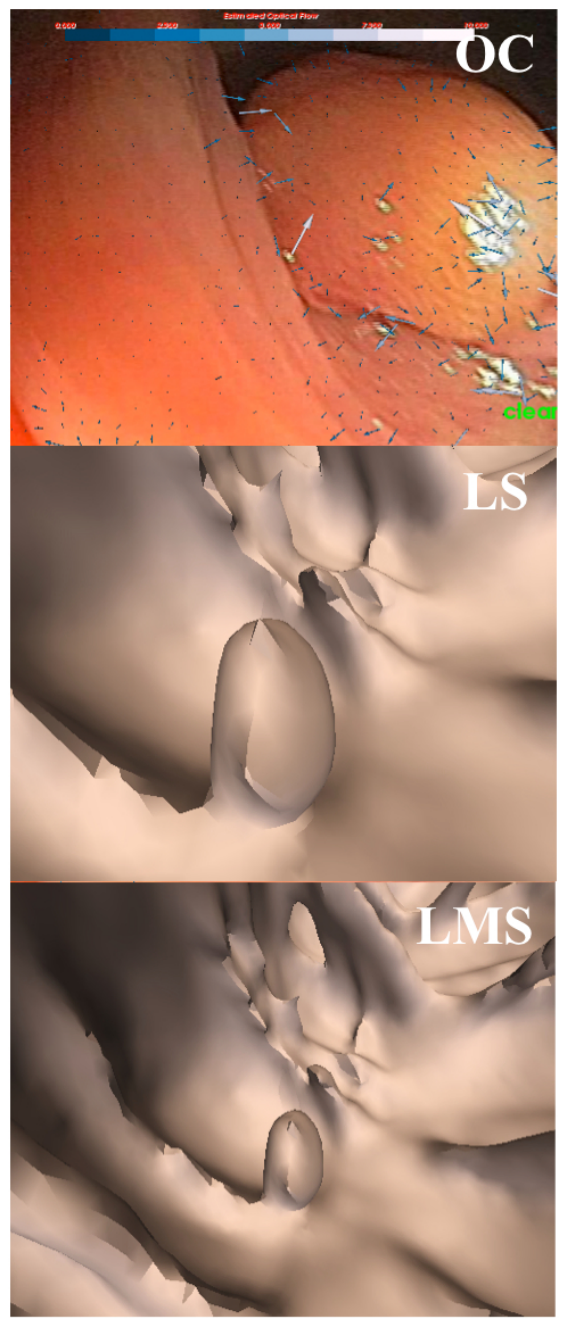

(b)
Frame. 410

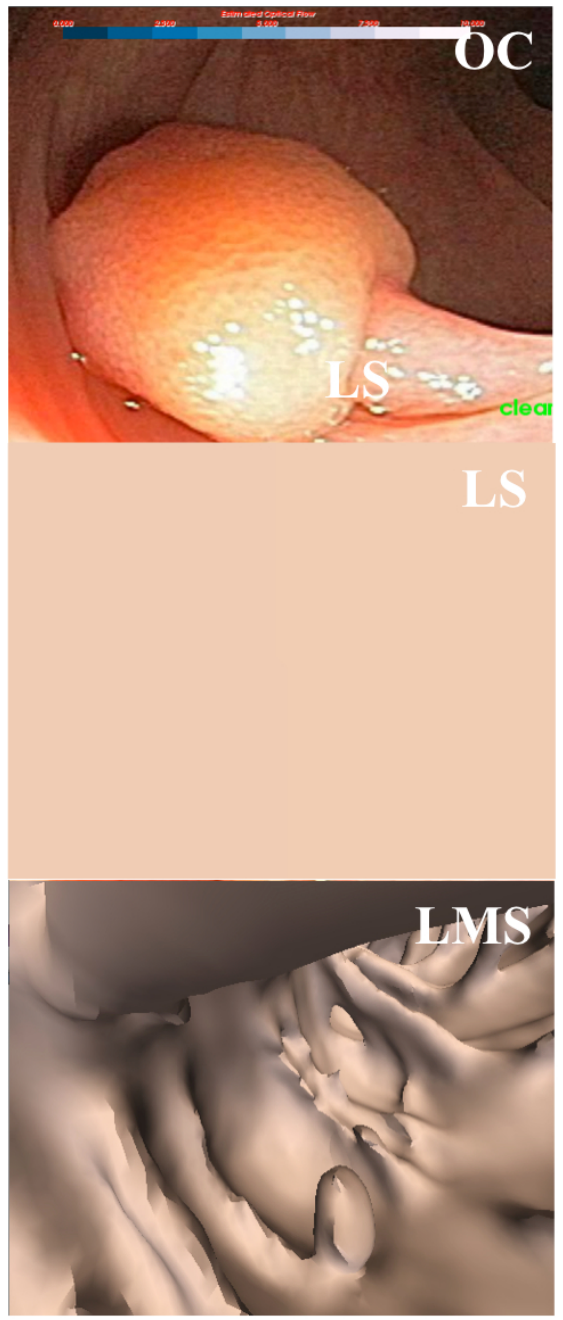

(c)

Figure 6. Comparison of LMS and LS tracking results in frame 50 (left column), 240 (center column), and 410 (right column) using a transverse colon image sequence. Here, LS estimator-based method failed after frame 410, while LMS tracks the full sequence(1316 frames).

of the LS-based method happens at frame 410, due to the colonoscope touching the polyp. The LMS-based method tracks the entire sequence. For the first 100 images, both LMS and LS estimators perform well. Beyond this, the images diverge; as in experiment 2, the LS-based method tracks translation well, but not rotation, while the LMS-based method performs better on both. This leads the LS-based method to fail at frame 410, when it gets too close to the polyp.

\section{CONCLUSIONS AND FUTURE WORK}

In this paper, we have augmented our optical flow based colonoscopy tracking algorithm with a robust regression method, Least Median of Squares, to accurately estimate motion parameters. The regression method permits detection of outliers among the chosen feature points. This leads to better estimates of rotation and translation parameters. Accumulation errors are reduced, making it possible to track longer sequences. Three experiments were performed on clinical colonoscopy datasets to illustrate the accuracy and robustness of the LMS-based 
estimator over the LS-based estimator. The first experiment demonstrates better location accuracy through the LMS method, while the second and third experiments show a large improvement in the number of tracked frames, from 300 to 1310 , and 410 to 1316 , respectively.

However, blurry images occur in the sequences when the colonoscope touches the colon wall or is immersed in fluid during surgery. The optical flow fields of such images are questionable because of the lack of texture and geometry information. In such situations, the LMS method can also fail. Thus, it is necessary to skip these problematic frames and reset the tracking pipeline to obtain more reliable flow fields, prior to estimating motion parameters using the LMS approach. We are currently investigating these issues.

\section{REFERENCES}

1. NCI, "A snapshot of colorectal cancer," $2002 . \quad$ National Cancer Institute, http://planning.cancer.gov/disease/Colorectal-Snapshot.pdf.

2. D. Nain, S. Haker, W. Grimson, E. C. Jr., W. M. Wells, H. Ji, R. Kikinis, and C. F. Westin, "Intrapatient prone to supine colon registration for synchronized colonoscopy," in Proceedings of 5th International Conference on Medical Image Computing and Computer-Assisted Intervention, pp. 573-580, 2002.

3. A. E. Kaufman, S. Lakare, K. Kreeger, and I. Bitter, "Virtual colonoscopy," Communications of the $A C M$ 48(2), pp. 37-41, 2005.

4. P. C. I. Bricault, G. Ferretti, "Multi-level strategy for computer-assisted transbronchial biopsy," in Proceedings of 1th International Conference on Medical Image Computing and Computer-Assisted Intervention, pp. 161-268, 1998.

5. L. Rai, S. A. Merritt, and W. E. Higgins, "Real-time image-based guidance method for lung-cancer assessment," in Proceedings of IEEE Conference on Computer Vision and Pattern Recognition, pp. 2437-2444, 2006.

6. D. Deguchi, K. Mori, Y. Suenaga, J. Hasegawa, J. Toriwaki, H. T. Batake, and H. Natori, "New image similarity measure for bronchoscope tracking based on image registration," in Proceedings of 6th International Conference on Medical Image Computing and Computer-Assisted Intervention, pp. 399-406, 2003.

7. J. Nagao, K. Mori, T. Enjouji, and D. Deguchi, "Fast and accurate bronchoscope tracking using image registration and motion prediction," in Proceedings of 7th International Conference on Medical Image Computing and Computer-Assisted Intervention, pp. 551-558, 2004.

8. K. Mori, D. Deguchi, K. Akiyama, T. Kitasaka, C. R. M. Jr., Y. Suenaga, H. Takabatake, M. Mori, and H. Natori, "Hybrid bronchoscope tracking using a magnetic tracking sensor and image registration," in Proceedings of 8th International Conference on Medical Image Computing and Computer-Assisted Intervention, pp. 543-555, 2005.

9. F. Deligianni, A. Chung, and G. Z. Yang, "Non-rigid 2d-3d registration with catheter tip em tracking for patient specific bronchoscope simulation," in Proceedings of 9th International Conference on Medical Image Computing and Computer-Assisted Intervention, pp. 281-288, 2006.

10. W. E. Higgins, J. P. Helferty, K. Lu, S. A. Merritt, L. Rai, and K.-C. Yu, "3d ct-video fusion for image-guided bronchoscopy," Computerized Medical Imaging and Graphics 32, pp. 159-173, 2007.

11. J. Liu, K. Subramanian, T. Yoo, and R. Uitert, "A stable optic-flow based method for tracking colonoscopy images," in Proc. of Mathematical Methods in Biomedical Image Analysis, 2008. June 27-28, Anchorage, Alaska.

12. F. R. Hampel, E. M. Ronchetti, P. J. Rousseeuw, and W. A. Stahel, Robust Statistics: The Approach Based on Influence Functions, Wiley-Interscience, first ed., 2005.

13. P. J. Rousseeuw and A. M. Leroy, Robust Regression and Outlier Detection, Wiley, first ed., 1987.

14. P. Meer, D. Mintz, A. Rosenfeld, and D. Y. Kim, "Robust regression methods for computer vision: a review," International Journal of Computer Vision 6(1), pp. 59-70, 1991.

15. C. V. Stewart, "Robust parameter estimation in computer vision," SIAM Reviews 41, pp. 513-537, 1999.

16. M. J. Black and P. Anadan, "The robust estimation of multiple motions: Parametric and piecewise-smooth flow fields," Computer Vision and Image Understanding 63(1), pp. 75-104, 1996. 
17. Z. Zhang and O. D. Faugeras, "Determining motion from 3d line segment matches: a comparative study," Image Vision Computing 9(1), pp. 10-19, 1991.

18. R. Hartley and A. Zisserman, Multiple View Geometry in Computer Vision, Cambridge University Press, second ed., 2004.

19. M. A. Fischler and R. C. Bolles, "Random sample consensus: a paradigm for model fitting with applications to image analysis and automated cartography," Communications of the ACM 24(6), pp. 381-395, 1981.

20. B. Horn and B. Schunck, "Determining optical flow," Artificial Intelligence 17(3), pp. 185-203, 1981.

21. P. J. Rousseeuw, "Least median of squares regression," Journal of the American Statistical Association 79(388), pp. 871-880, 1984. 